\title{
BMJ Open Study protocol for efficacy and safety of steroid-containing mouthwash to prevent chemotherapy-induced stomatitis in women with breast cancer: a multicentre, open-label, randomised phase 2 study
}

\begin{abstract}
Sayaka Kuba (D) , ${ }^{1}$ Kosho Yamanouchi, ${ }^{2}$ Megumi Matsumoto, ${ }^{3}$ Shigeto Maeda, ${ }^{2}$ Toshiko Hatachi, ${ }^{4}$ Sakiko Soutome,${ }^{5}$ Yumiko Kawashita, ${ }^{5}$ Michi Morita, ${ }^{1}$ Chika Sakimura, ${ }^{6}$ Eiko Inamasu, ${ }^{7}$ Kenichiro Shibata, ${ }^{4}$ Ryota Otsubo, ${ }^{3}$ Hiroshi Yano, ${ }^{3}$ Seiichi Nose, ${ }^{8}$ Junya Miyamoto, ${ }^{9}$ Shuntaro Sato, ${ }^{9}$ Kengo Kanetaka, ${ }^{1}$ Hideki Taniguchi, ${ }^{4}$ Masahiro Umeda, ${ }^{10}$ Takeshi Nagayasu, ${ }^{3}$ Susumu Eguchi ${ }^{1}$
\end{abstract}

To cite: Kuba S, Yamanouchi K, Matsumoto M, et al. Study protocol for efficacy and safety of steroid-containing mouthwash to prevent chemotherapy-induced stomatitis in women with breast cancer: a multicentre open-label, randomised phase 2 study. BMJ Open 2020;10:e033446. doi:10.1136/ bmjopen-2019-033446

- Prepublication history and additional material for this paper are available online. To view these files, please visit the journal online (http://dx.doi. org/10.1136/bmjopen-2019033446).

Received 05 August 2019 Revised 29 November 2019 Accepted 17 December 2019

Check for updates

(C) Author(s) (or their employer(s)) 2020. Re-use permitted under CC BY-NC. No commercial re-use. See rights and permissions. Published by BMJ.

For numbered affiliations see end of article.

Correspondence to

Dr Sayaka Kuba;

skuba@nagasaki-u.ac.jp

\section{ABSTRACT}

Introduction Stomatitis is a frequent adverse event in patients undergoing chemotherapy for breast cancer. Stomatitis can hamper oral nutrition resulting in malnutrition, reduce quality of life and introduce the need for dose reductions and interruption of chemotherapy; however, there is currently no standard approach for preventing chemotherapy-induced stomatitis. We aimed to assess the safety and efficacy of a dexamethasone-based elixir mouthwash for preventing chemotherapy-induced stomatitis in patients with early breast cancer. Methods and analysis In this multicenter, randomised, controlled phase 2 trial, we will randomly assign 120 women with early breast cancer undergoing chemotherapy to use of a dexamethasone-based elixir or standard oral care, to compare their preventive effects on chemotherapy-induced stomatitis. Patients will be assigned in a 1:1 ratio. Patients in the intervention group will receive chemotherapy, oral care and a dexamethasone-based elixir $(10 \mathrm{~mL} 0.1 \mathrm{mg} / \mathrm{mL}$; swish for $2 \mathrm{~min}$ and spit, four times daily for 9 weeks), and patients in the control group will receive chemotherapy and oral care. The primary endpoint is the difference in incidence of stomatitis between the two groups. The sample size allows for the detection of a minimum difference of $20 \%$ in the incidence of stomatitis between the two groups. Secondary endpoints are severity of stomatitis, duration of stomatitis, completion rate of chemotherapy and adverse events. Ethics and dissemination All participants signed a written consent form, and the study protocol has been reviewed and approved by the Clinical Research Review Board of Nagasaki University (CRB7180001).

Trial registration number UMIN Clinical Trials Registry (UMIN000030489).

\section{INTRODUCTION}

Stomatitis can be painful, affect the patient's nutrition and quality of life, and have a

\section{Strengths and limitations of this study}

This is a multicenter, randomised, controlled, open-label phase 2 trial to evaluate the effect of a dexamethasone-based elixir on preventing chemotherapy-induced stomatitis.

- The study aims is to generate evidence for the efficacy of a dexamethasone-based elixir mouthwash for preventing chemotherapy-induced stomatitis in patients with early breast cancer.

- All women will receive a medical check-up by a dentist, and stomatitis will therefore be correctly diagnosed allowing the earlier identification of oral adverse events.

- The incidence of stomatitis is determined from the patients' records and does not require an additional medical examination by the dentist.

No placebo drug is used in the control group.

significant economic impact. ${ }^{1}$ Stomatitis occurs in approximately $20 \%-40 \%$ of patients receiving conventional chemotherapy, $80 \%$ of patients receiving high-dose chemotherapy as conditioning for haematopoietic stem cell transplantation, and in nearly all patients receiving head and neck radiation therapy. ${ }^{1}$ Masuda et al reported a preventive effect of rebamipide in a randomised, multicenter phase 2 study of breast cancer patients with chemotherapy-induced stomatitis treated with 5-fluorouracil, epirubicin and cyclophosphamide, or with docetaxel and cyclophosphamide (TC), ${ }^{2}$ and showed that about $60 \%$ of patients in both groups developed stomatitis. 
The pathogenesis of stomatitis is multifactorial and complex. Lalla et al proposed that stomatitis occurred via five stages: (1) initiation of tissue injury, (2) upregulation of inflammation via generation of messenger signals, (3) signalling and amplification, (4) ulceration and inflammation and (5) healing. ${ }^{3}$ This theory proposes that proinflammatory cytokines, produced mainly by macrophages, injure mucosal cells and activate molecular pathways that amplify mucosal injury. Subsequent inflammatory cell infiltrates are associated with mucosal ulceration, and excessive inflammation seems to exacerbate stomatitis.

Several studies have attempted to prevent chemotherapyinduced stomatitis. Palifermin reduced the duration and severity of stomatitis after intensive chemotherapy and radiotherapy in patients with haematological cancer. ${ }^{4}$ Palifermin has therefore been approved by the US Food and Drug Administration and the European Medicines Agency for stomatitis patients with haematological malignancies. Oral cryotherapy prevented stomatitis in patients receiving bolus doses of 5-fluorouracil and haematopoietic stem cell transplantation. ${ }^{5}$ Rebamipide is an internal-use mucosal protectant, and gargling with rebamipide has been reported to prevent stomatitis in patients with head and neck cancer who are receiving chemoradiotherapy. ${ }^{67}$ However, recommendations for preventing chemotherapy-induced stomatitis in patients with breast cancer are lacking.

Oral care protocols are used to prevent stomatitis in all cancer treatment modalities. ${ }^{1}$ Saforis (glutamine) is reportedly effective for preventing and treating stomatitis in patients with histopathologically confirmed breast cancer receiving anthracycline-based chemotherapy. ${ }^{8}$ However, internal rebamipide showed no efficacy in preventing chemotherapy-induced stomatitis in breast cancer patients. ${ }^{2}$ The Multinational Association of Supportive Care in Cancer (MASCC) MASCC/ISOO Mucositis Guidelines 2019 Update recommends photobiomodulation, parenteral or oral glutamine, and benzydamine mouthwash in patients undergoing haematopoietic stem cell transplantation and patients with head and neck cancer receiving radiotherapy with or without concomitant chemotherapy. ${ }^{9-12}$ However, there is currently no recommendation for the prevention of stomatitis in breast cancer patients receiving chemotherapy without multi-agent combination oral care protocols.

Everolimus is an oral mammalian target of rapamycin (mTOR) inhibitor used to treat hormone receptorpositive/human epidermal growth factor receptor 2-negative breast cancer. Although the addition of an mTOR inhibitor to an aromatase inhibitor has been shown to prolong progression-free survival compared with an aromatase inhibitor alone, stomatitis is a frequent adverse effect of mTOR inhibitors. ${ }^{13}$ The SWISH trial compared the prophylactic use of dexamethasone oral solution with historical controls from the BOLERO-2 trial, and showed that dexamethasone substantially reduced the incidence and severity of stomatitis in patients receiving an mTOR inhibitor by $40 \%$ (oral mucositis rate: $21 \%$ in the SWISH trial vs $61 \%$ in the BOLERO-2 trial). ${ }^{14}$ The pathogenesis of stomatitis is therefore thought to involve excessive

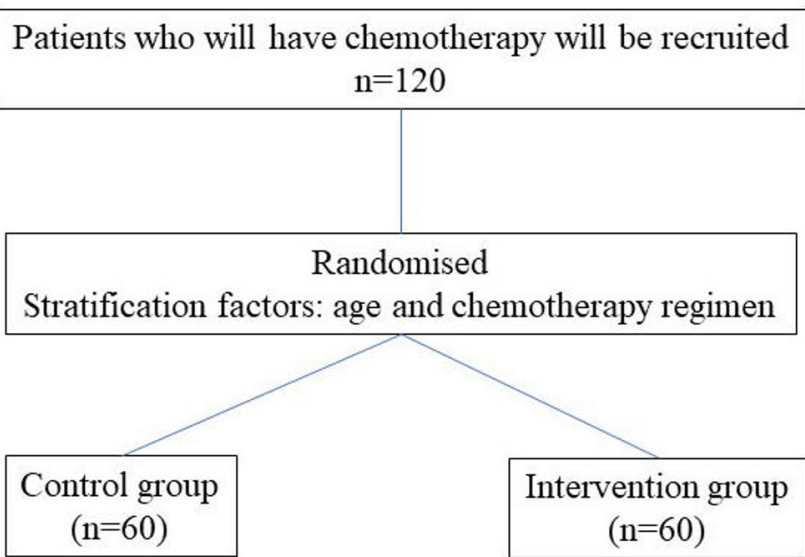

Figure 1 Flowchart of participants.

inflammation and can be treated by topical dexamethasone ointment. A prednisolone-containing mouthwash was also recently reported to reduce the incidence of stomatitis in breast cancer patients who developed grade 2-3 oral mucositis during the previous treatment cycle. ${ }^{15}$ However, the study was not a randomised controlled trial and did not report the adverse events in detail.

The current randomised, controlled, phase 2 trial aims to assess the safety and efficacy of a dexamethasone-based elixir mouthwash for preventing chemotherapy-induced stomatitis in patients with early breast cancer.

\section{METHODS AND ANALYSIS Methods/design}

This is a multicenter, randomised, controlled, open-label phase 2 trial to evaluate the effect of a dexamethasonebased elixir on preventing chemotherapy-induced stomatitis. The patient flowchart is shown in figure 1.

\section{Eligibility criteria}

Eligible patients are women with histologically confirmed invasive breast cancer, aged 20-80 years, with a performance status of $0-1$, scheduled to receive epirubicin and cyclophosphamide or TC. Patients must have a normal oral mucosa at baseline. Patients with distant metastasis, prior treatment for breast cancer, unsuitable dentures, or who are pregnant or breast-feeding will be excluded.

\section{Recruitment, setting and informed consent}

Patients are being recruited from Nagasaki University, National Hospital Organization Nagasaki Medical Center and Japanese Red Cross Nagasaki Genbaku Hospital from December 2018. Patients are recruited when their chemotherapy regimen is explained. All women are screened again to determine their eligibility and are provided with a participant information sheet and asked to provide their written consent. A copy of the patient consent form is available as online supplementary file 1. Participants have the right to withdraw from the study at any time without having to give a reason. 
Table 1 Time of data collection

\begin{tabular}{llllll} 
& \multicolumn{2}{l}{ Collection time } & & \\
\cline { 2 - 5 } & Booking & Day 1 of cycle 1 & Day 1 of cycle 2 & Day 1 of cycle 3 & Day 1 of cycle 4 \\
\hline $\begin{array}{l}\text { Consultation with a dentist } \\
\text { Check infection and stomatitis }\end{array}$ & $\circ$ & 0 & 0 & 0 \\
Collect patient's report & - & - & 0 & 0 & 0 \\
Adverse events & - & - & $\circ$ & 0 & $\circ$ \\
Number of bacteria (saliva) & $\circ$ & & $\circ$ & $\circ$ & 0 \\
\hline
\end{tabular}

\section{Randomisation}

The first researcher (SK) assigns women to the intervention or control groups using a centralised, remote computer-generated randomiser. Stratification factors are age and chemotherapy regimen. There will be an intervention-to-control ratio of 1:1. All data, including electronic and hardcopies, will be destroyed 5 years after the completion of the study.

\section{Treatment and assessment schedule}

The study period runs from day 1 of the first cycle of chemotherapy until day 1 of the fourth cycle of chemotherapy. Beginning on day 1 of the first cycle of chemotherapy, patients in the intervention group receive chemotherapy plus $10 \mathrm{~mL}$ of a dexamethasone-based elixir $(0.1 \mathrm{mg} / \mathrm{mL}$; swish for $2 \mathrm{~min}$ and spit, four times daily for 9 weeks). Other drugs and mouthwashes without dexamethasone will be allowed in both groups. The datacollection schedule is shown in table 1 .

In this study, all women in both groups will receive standard oral care and a medical examination by a dentist and dental hygienist. First, the dentist performs oral and dental screening, including a radiographical examination, and measures the number of bacteria in the saliva before chemotherapy. The dentist then checks for dental caries and stomatitis every 3 weeks on chemotherapy day. Patients also receive toothbrushing instructions and professional mechanical tooth cleaning before and during chemotherapy. If an intraoral infection is detected in any patient in the intervention group, use of the dexamethasone-based elixir is stopped. Physicians check for any adverse events (both haematological and non-haematological toxicities) using the Common Terminology Criteria for Adverse Events (CTCAE) V.4.0. Patients will record the daily incidence of anorexia, stomatitis, food intake and dysgeusia, and the frequency of mouthwash use (only the intervention group) and of intraoral steroid ointment use (if present) (figure 2). Because there is currently no standard intervention without oral care to prevent chemotherapy-induced stomatitis, all patients are allowed to use any medicines or supplements for preventing mucositis. As noted above, patients are required to record the use of intraoral steroid ointment for the treatment of stomatitis.

\section{Trial endpoints}

Primary endpoint

The primary endpoint is the incidence of all-grade stomatitis according to patients' records after 9 weeks.

\begin{tabular}{|c|c|c|c|c|c|c|c|c|c|c|c|c|c|c|c|c|c|c|c|c|c|}
\hline $\begin{array}{ll}\text { Adverse events } & \text { Chemotherapy day } \\
\end{array}$ & 1 & 2 & 3 & 4 & 5 & 6 & 7 & 8 & 9 & 10 & 11 & 12 & 13 & 14 & 15 & 16 & 17 & 18 & 19 & 20 & 21 \\
\hline \multirow{3}{*}{$\begin{array}{l}\text { Anorexia } \\
\text { No } \\
\text { Yes, but no decrease in food intake } \\
\text { Decrease in food intake }\end{array}$} & & & & & & & & & & & & & & & & & & & & & \\
\hline & & & & & & & & & & & & & & & & & & & & & \\
\hline & & & & & & & & & & & & & & & & & & & & & \\
\hline \multirow{2}{*}{$\begin{array}{l}\text { Stomatitis } \\
\text { Yes } \\
\text { No }\end{array}$} & & & & & & & & & & & & & & & & & & & & & \\
\hline & & & & & & & & & & & & & & & & & & & & & \\
\hline \multirow{3}{*}{$\begin{array}{l}\text { Pain of mouth } \\
\text { No } \\
\text { Yes, but no problems with food intake } \\
\text { I have problems with food intake }\end{array}$} & & & & & & & & & & & & & & & & & & & & & \\
\hline & & & & & & & & & & & & & & & & & & & & & \\
\hline & & & & & & & & & & & & & & & & & & & & & \\
\hline \multirow{3}{*}{$\begin{array}{l}\text { Dysgeusia } \\
\text { No } \\
\text { Yes, but no change in diet } \\
\text { Yes, change in diet or ageusia }\end{array}$} & & & & & & & & & & & & & & & & & & & & & \\
\hline & & & & & & & & & & & & & & & & & & & & & \\
\hline & & & & & & & & & & & & & & & & & & & & & \\
\hline $\begin{array}{l}\text { Number of gargling sessions } \\
\text { (intervention group) }\end{array}$ & & & & & & & & & & & & & & & & & & & & & \\
\hline $\begin{array}{l}\text { Number of times topical dexamethasone } \\
\text { ointment was applied (if used) }\end{array}$ & & & & & & & & & & & & & & & & & & & & & \\
\hline
\end{tabular}

Figure 2 Patients' records. Patients will record the daily incidences of anorexia, stomatitis, food intake and dysgeusia, and the frequency of mouthwash use (only the intervention group) and of intraoral steroid ointment use (if present). 


\section{Secondary endpoints}

1. Incidence of stomatitis by grade in the intervention and control groups.

2. Duration of stomatitis in the intervention and control groups.

3. Completion rate of chemotherapy.

4. Relative dose intensity of chemotherapy.

5. Frequency of mouthwash use per day in the intervention group.

6. Adverse events according to CTCAE V.4.0.

7. Incidence of intraoral infections.

\section{Safety monitoring}

Any severe adverse events must be reported to the principal investigator. The principal investigator will then gather all information regarding these adverse events reported by the investigators from each institution and report them to the Clinical Research Review Board at Nagasaki University.

\section{Sample size}

On the basis of a previous study, we predicted the incidence of mucositis to be $50 \%$ in the control group and $30 \%$ in the intervention group. ${ }^{21516}$ We calculated that the trial would therefore need to include 54 patients in each group with a beta level of 0.1 and an alpha level of 0.2 (one-sided) using the $\chi^{2}$ test. Considering a $10 \%$ potential withdrawal rate, we therefore planned to enrol 120 patients (60 per group). The treatment-to-control ratio was 1:1.

\section{Data management}

Data will be collected using case report forms. Data will be provided with a study code, and deidentified data will be used for the analyses. All hardcopies will be deidentified using a study code and stored securely in the hospital setting. All data will be discarded 5 years after completion of the study.

\section{Statistical analyses}

Analyses of the primary and secondary outcomes will be based on an intention-to-treat analysis, which will include withdrawal from the study. The patients' clinicopathological features will be compared between the intervention and control groups. Variables will be described as frequencies for categorical variables and median and interquartile ranges for quantitative variables. Associations between variables will be assessed using Fisher's exact test, with Wilcoxon's rank sum test used for quantitative variables. All statistical analyses will be performed using EZR software (Saitama Medical Center, Jichi Medical University, Japan).

\section{Study status}

Patients were first enrolled in December 2018. Fifty-five patients had been recruited as of April 2019, and recruitment is still ongoing.

\section{Patient and public involvement statements}

This research was carried out without patient or public involvement. Neither patients nor the public were involved in the development of the research question, study design or implementation of this trial. Patients will not be invited to develop patient-relevant outcomes or interpret the results, or to participate in the writing or editing of the final manuscript for readability or accuracy. Because the interventions in our study are routine procedures during clinical work, the burden of the intervention is assessed by the patients themselves.

\section{ETHICS AND DISSEMINATION}

All participants signed a written consent form. The findings will be disseminated through scientific and professional conferences, and in peer-reviewed journals.

Author affiliations

${ }^{1}$ Surgery, Nagasaki University School of Medicine Graduate School of Biomedical Sciences, Nagasaki, Japan

${ }^{2}$ Surgery, National Hospital Organisation Nagasaki Medical Center, Omura, Nagasaki, Japan

${ }^{3}$ Surgical Oncology, Nagasaki University School of Medicine Graduate School of Biomedical Sciences, Nagasaki, Japan

${ }^{4}$ Surgery, Japanese Red Cross Nagasaki Atomic Bomb Hospital, Nagasaki, Japan

${ }^{5}$ Oral Management Center, Nagasaki University Hospital, Nagasaki, Japan

${ }^{6}$ Surgery, Nagasaki Harbor Medical Center City Hospital, Nagasaki, Japan

${ }^{7}$ Hakujujikai Sasebo Chuo Hospital, Sasebo, Nagasaki, Japan

${ }^{8}$ Pharmacy, Nagasaki University Hospital, Nagasaki, Japan

${ }^{9}$ Clinical Research Center, Nagasaki University Hospital, Nagasaki, Japan

${ }^{10} \mathrm{Clinical}$ Oral Oncology, Nagasaki University School of Medicine Graduate School of Biomedical Sciences, Nagasaki, Japan

Correction notice The article has been corrected since it is published. The authorname, Sakiko Soutome has been updated.

Acknowledgements The authors thank Ms. Yamamura and Ms. Haraguchi for creating and managing the database. We also thank Mark Abramovitz, $\mathrm{PhD}$, and Susan Furness, PhD, from Edanz Group (www.edanzediting.com/ac) for editing a draft of this manuscript.

Contributors SK, KY, MM, SM and TH are responsible for conceiving and designing the trial, planning data analysis and collecting data, and are incharge of patient recruitment and treatment. MM, CS, El, KS, RO and HY will participate in data collection and are incharge of patient recruitment and treatment. SN is responsible for preparing the dexamethasone-based elixir mouthwash. SS and YK are responsible for dental checkups and judging stomatitis and oral adverse events. JM and SS are responsible for the sample size and allocation. KK, HT, MU, TN and SE are responsible for planning the data analysis and analysing the data resulting from the trial. SK is responsible for screening the patients and data monitoring with attending the physician. SK, JM and SS will access the final trial dataset and analyse the data. All authors have contributed to and approved the final version of this manuscript for publication.

Funding Funding for this research was obtained through Project Mirai Cancer Research Grants and grants from the Nagasaki-ken Medical Association.

Competing interests None declared.

Patient consent for publication Not required.

Ethics approval This study was approved by the Clinical Research Review Board of Nagasaki University, Japan. Any changes that need to be made to the trial protocol will be communicated to all investigators and ethics committees, and to the trial registry. The protocol (version 1) has been designed and will be conducted in accordance with the Declaration of Helsinki (1964) and the Clinical Trial Act (2018).

Provenance and peer review Not commissioned; externally peer reviewed.

Open access This is an open access article distributed in accordance with the Creative Commons Attribution Non Commercial (CC BY-NC 4.0) license, which permits others to distribute, remix, adapt, build upon this work non-commercially, and license their derivative works on different terms, provided the original work is properly cited, appropriate credit is given, any changes made indicated, and the use is non-commercial. See: http://creativecommons.org/licenses/by-nc/4.0/.

ORCID iD 
Sayaka Kuba http://orcid.org/0000-0002-0225-099X

\section{REFERENCES}

1 Lalla RV, Bowen J, Barasch A, et al. MASCC/ISOO clinical practice guidelines for the management of mucositis secondary to cancer therapy. Cancer 2014;120:1453-61.

2 Masuda N, Nishimura S, Ohno S, et al. Preventive effect of rebamipide on chemotherapy-induced oral mucositis in breast cancer patients: a randomized multi-center phase II study. MASCC/ ISOO annual meeting 2014

3 Lalla RV, Sonis ST, Peterson DE. Management of oral mucositis in patients who have cancer. Dent Clin North Am 2008:52:61-77.

4 Spielberger R, Stiff P, Bensinger W, et al. Palifermin for oral mucositis after intensive therapy for hematologic cancers. N Engl J Med 2004;351:2590-8.

5 Raber-Durlacher JE, von Bültzingslöwen I, Logan RM, et al. Systematic review of cytokines and growth factors for the management of oral mucositis in cancer patients. Support Care Cancer 2013;21:343-55.

6 Chaitanya B, Pai KM, Yathiraj PH, et al. Rebamipide gargle in preventive management of chemo-radiotherapy induced oral mucositis. Oral Oncol 2017;72:179-82.

7 Yokota T, Ogawa T, Takahashi S, et al. Efficacy and safety of rebamipide liquid for chemoradiotherapy-induced oral mucositis in patients with head and neck cancer: a multicenter, randomized, double-blind, placebo-controlled, parallel-group phase II study. BMC Cancer 2017;17:314.

8 Peterson DE, Jones JB, Petit RG. Randomized, placebo-controlled trial of Saforis for prevention and treatment of oral mucositis in breast cancer patients receiving anthracycline-based chemotherapy. Cancer 2007;109:322-31.

9 Zadik Y, Arany PR, Fregnani ER, et al. Mucositis Study group of the multinational association of supportive care in Cancer/International
Society of oral oncology (MASCC/ISOO). systematic review of photobiomodulation for the management of oral mucositis in cancer patients and clinical practice guidelines. Support Care Cancer 2019;27:3969-83.

10 Yarom N, Hovan A, Bossi P, et al. Mucositis Study group of the multinational association of supportive care in cancer / International Society of oral oncology (MASCC/ISOO). systematic review of natural and miscellaneous agents for the management of oral mucositis in cancer patients and clinical practice guidelines. Part 1: vitamins, minerals, and nutritional supplements. Support Care Cancer 2019;27:3997-4010.

11 Ariyawardana A, Cheng KKF, Kandwal A, et al. Mucositis Study group of the multinational association of supportive care in Cancer/ International Society for oral oncology (MASCC/ISOO). systematic review of anti-inflammatory agents for the management of oral mucositis in cancer patients and clinical practice guidelines. Support Care Cancer 2019;27:3985-95.

12 Hong CHL, Gueiros LA, Fulton JS, et al. Mucositis Study group of the multinational association of supportive care in Cancer/ International Society for oral oncology (MASCC/ISOO). systematic review of basic oral care for the management of oral mucositis in cancer patients and clinical practice guidelines. Support Care Cancer 2019;27:3949-67.

13 Baselga J, Campone M, Piccart M, et al. Everolimus in postmenopausal hormone-receptor-positive advanced breast cancer. N Engl J Med 2012;366:520-9.

14 Rugo HS, Seneviratne L, Beck JT, et al. Prevention of everolimusrelated stomatitis in women with hormone receptor-positive, HER2negative metastatic breast cancer using dexamethasone mouthwash (SWISH): a single-arm, phase 2 trial. Lancet Oncol 2017;18:654-62.

15 Cidon EU. Chemotherapy induced oral mucositis: prevention is possible. Chin Clin Oncol 2018;7:6.

16 Del Mastro L, De Placido S, Bruzzi P, et al. Fluorouracil and dose-dense chemotherapy in adjuvant treatment of patients with early-stage breast cancer: an open-label, $2 \times 2$ factorial, randomised phase 3 trial. Lancet 2015;385:1863-72. 